\title{
Strategic Anti-SARS-CoV-2 Serology Testing in a Low Prevalence Setting: The COVID-19 Contact (CoCo) Study in Healthcare Professionals
}

\author{
Georg M. N. Behrens · Anne Cossmann · Metodi V. Stankov • \\ Bianca Schulte · Hendrik Streeck · Reinhold Förster · Berislav Bosnjak • \\ Stefanie Willenzon • Anna-Lena Boeck • Anh Thu Tran • \\ Thea Thiele - Theresa Graalmann • Moritz Z. Kayser • Anna Zychlinsky Scharff • \\ Christian Dopfer · Alexander Horke · Isabell Pink · Torsten Witte • \\ Martin Wetzke · Diana Ernst · Alexandra Jablonka (D) · \\ Christine Happle
}

Received: July 31, 2020 / Published online: September 4, 2020

(C) The Author(s) 2020

\section{ABSTRACT}

Background: Serology testing is explored for epidemiological research and to inform

Georg M. N. Behrens, Anne Cossmann, Alexandra Jablonka and Christine Happle contributed equally to this work.

Digital Features To view digital features for this article go to https://doi.org/10.6084/m9.figshare.12841166.

Electronic supplementary material The online version of this article (https://doi.org/10.1007/s40121020-00334-1) contains supplementary material, which is available to authorized users.

G. M. N. Behrens ( $₫)$. A. Cossmann .

M. V. Stankov · T. Thiele · T. Graalmann ·

T. Witte · D. Ernst . A. Jablonka

Department for Rheumatology and Clinical

Immunology, Hannover Medical School, Hannover,

Germany

e-mail: behrens.georg@mh-hannover.de

G. M. N. Behrens · A. Cossmann · M. V. Stankov ·

M. Wetzke · A. Jablonka

German Center for Infection Research (DZIF),

Partner Site Hannover-Braunschweig, Brunswick, Germany

G. M. N. Behrens

CiiM, Centre for Individualised Infection Medicine,

Hannover, Germany individuals after suspected infection. During the coronavirus disease 2019 (COVID-19) pandemic, frontline healthcare professionals (HCP) may be at particular risk for infection. No longitudinal data on functional seroconversion in HCP in regions with low COVID-19 prevalence and low pre-test probability exist.

Methods: In a large German university hospital, we performed weekly questionnaire assessments and anti-severe acute respiratory syndrome coronavirus 2 (SARS-CoV-2) immunoglobulin G (IgG) measurements with various commercial tests, a novel surrogate virus neutralisation test, and a neutralisation assay using live SARS-CoV-2.

B. Schulte $\cdot$ H. Streeck

Institute of Virology, University Hospital, University of Bonn, Bonn, Germany

B. Schulte $\cdot$ H. Streeck

German Center for Infection Research (DZIF),

Partner Site Bonn-Köln, Bonn, Germany

R. Förster · B. Bosnjak · S. Willenzon Institute for Immunology, Hannover Medical School, Hannover, Germany

R. Förster

Cluster of Excellence RESIST (EXC 2155), Hannover

Medical School, Hannover, Germany 
Results: From baseline to week 6, 1080 screening measurements for anti-SARS CoV-2 (S1) IgG from 217 frontline HCP (65\% female) were performed. Overall, $75.6 \%$ of HCP reported at least one symptom of respiratory infection. Self-perceived infection probability declined over time (from mean $20.1 \%$ at baseline to $12.4 \%$ in week 6 , $p<0.001$ ). In sera of convalescent patients with PCR-confirmed COVID-19, we measured high anti-SARS-CoV-2 IgG levels, obtained highly concordant results from enzyme-linked immunosorbent assays (ELISA) using e.g. the spike 1 (S1) protein domain and the nucleocapsid protein (NCP) as targets, and confirmed antiviral neutralisation. However, in HCP the cumulative incidence for anti-SARS-CoV-2 (S1) IgG was $1.86 \%$ for positive and $0.93 \%$ for equivocal positive results over the study period of 6 weeks. Except for one HCP, none of the eight initial positive results were confirmed by alternative serology tests or showed in vitro neutralisation against live SARS-CoV-2. The only true seroconversion occurred without symptoms and mounted strong functional humoral immunity. Thus, the confirmed cumulative incidence for neutralizing anti-SARS-CoV-2 IgG was $0.47 \%$.

\author{
A.-L. Boeck · A. Thu Tran \\ Department for Neurology, Hannover Medical \\ School, Hannover, Germany \\ T. Graalmann \\ TWINCORE, Centre for Experimental and Clinical \\ Infection Research, Institute for Experimental \\ Infection Research, Hannover, Germany \\ M. Z. Kayser · I. Pink \\ Department for Pneumology, Hannover Medical \\ School, Hannover, Germany \\ A. Zychlinsky Scharff - C. Dopfer - M. Wetzke · \\ C. Happle \\ Department of Pediatric Pneumology, Allergology \\ and Neonatology, Hannover Medical School, \\ Hannover, Germany \\ A. Horke \\ Department of Cardiac, Thoracic, Transplantation \\ and Vascular Surgery, Hannover Medical School, \\ Hannover, Germany \\ C. Happle \\ German Center for Lung Research, Biomedical \\ Research in End Stage and Obstructive Lung \\ Disease/BREATH, Hannover, Germany
}

Conclusion: When assessing anti-SARS-CoV-2 immune status in individuals with low pre-test probability, we suggest confirming positive results from single measurements by alternative serology tests or functional assays. Our data highlight the need for a methodical serology screening approach in regions with low SARSCoV-2 infection rates.

Trial Registration: The study is registered at DRKS00021152.

Keywords: Coronavirus;

COVID-19;

Healthcare professionals; Humoral immunity; Infection; Pandemic; SARS-CoV-2; Serological testing; Virus

\section{Key Summary Points}

\section{Why carry out this study?}

The risk to healthcare professionals (HCP) of contracting COVID-19 in the workplace has been a pressing issue and no longitudinal studies in regions with a low prevalence of COVID-19 burden have been conducted so far.

More information on seroconversion is needed to help interpret individual serology test results.

We aimed to prospectively assess the validity of different serological testing systems in frontline HCP, to detect clinically silent seroconversions, and to determine the quality of systemic humoral immune responses.

\section{What was learned from the study?}

Over 6 weeks, the cumulative incidence for anti-SARS-CoV-2 (S1) IgG was 1.86\%. However, except for one HCP, none of the eight initial positive results were confirmed by alternative serology or functional tests. Thus, the confirmed cumulative incidence for neutralizing anti-SARS-CoV-2 IgG was $0.47 \%$. 
Our study supports the use of a two-step approach for determining humoral immune response against SARS-CoV-2. A positive result in a single measurement should be confirmed by alternative serology tests or functional assays.

\section{DIGITAL FEATURES}

This article is published with digital features to facilitate understanding of the article. You can access the digital features on the article's associated Figshare page. To view digital features for this article go to https://doi.org/10.6084/m9. figshare.12841166.

\section{INTRODUCTION}

Uncertain rates of asymptomatic infections have raised concerns about a potentially high rate of undiagnosed infections with severe acute respiratory syndrome coronavirus 2 (SARS-CoV2) $[1,2]$. Healthcare professionals (HCP) were shown to be at risk of infection during previous coronavirus outbreaks $[3,4]$. During the current pandemic, asymptomatic SARS-CoV-2 infection [5] and onward transmission of SARS-CoV-2 in HCP have been demonstrated [6, 7]. However, nosocomial spread to HCP depends on regional infection patterns $[1,8]$. In Wuhan, where the SARS-CoV-2 outbreak was first reported, the incidence of coronavirus disease 2019 (COVID19) was higher in HCP than the general public [9]. In contrast, studies from Spain and Belgium demonstrated SARS-CoV-2 infection rates of $6-30 \%$ irrespective of patient contact $[9,10]$, likely reflecting pandemic spread in the general population. Thus, both local infection dynamics and work place precautions against SARSCoV-2 transmission such as personal protection equipment (PPE) affect an HCP's risk of becoming infected.

SARS-CoV-2-specific B cell responses typically lead to detectable antibody titers and fully positive rates at about 18 days after the initial onset of symptoms [11]. Seroepidemiological studies can help to catalogue those who have been previously infected (including mild or subclinical infections) and may help identify atrisk populations [12]. Longitudinal analysis of humoral immunity is particularly valuable in persons at high risk for exposure such as HCP. We [13] and others [14] have demonstrated that the degree of humoral immune responses as assessed by enzyme-linked immunosorbent assay (ELISA) correlates with severity of COVID19. Consequently, it is important to explore whether asymptomatic SARS-CoV-2 infections also lead to detectable and functional antibody responses. Various in-house and commercial serological testing systems for SARS-CoV-2 specific immunoglobulins (Ig) to support clinical decision-making and epidemiological studies are currently on the market or being developed [15]. However, interpretation of individual anti-SARS-CoV-2 serology results in HCP and others depends not only on sensitivity and specificity of the testing systems but also on the regional prevalence of SARS-CoV-2 infections and the resulting pre-test probability of disease.

To further assess the validity of different serological testing systems in frontline HCP, we carried out the prospective COVID-19 Contact (CoCo) Study at Hannover Medical School, a large university hospital in Northern Germany. Our aims were (1) to obtain longitudinal data about the actual and self-perceived risk of infection, (2) to detect clinically silent seroconversions, (3) to assess the performance of serological testing systems (ELISAs and rapid test) detecting SARS-CoV-2 antibodies (total, IgG, immunoglobulin A (IgA)), and (4) to determine the quality of systemic humoral immune responses detected by ELISAs by employing a novel in vitro virus inhibition test and neutralisation assay using live virus.

\section{METHODS}

\section{Study Design, Enrolment and Follow-Up}

The CoCo study [13] is an ongoing prospective study which longitudinally monitors SARS- 


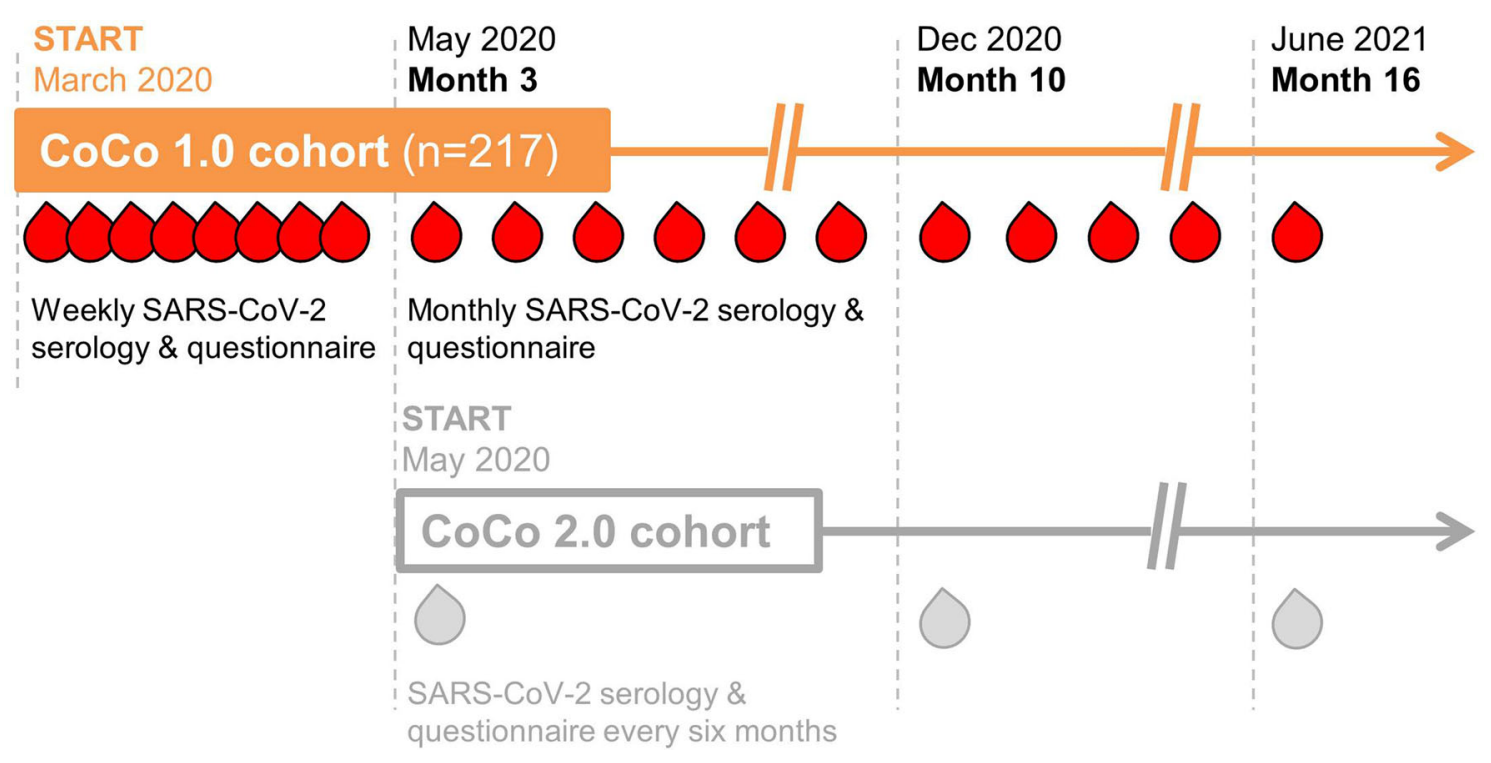

Fig. 1 Design of the CoCo study. The CoCo 1.0 cohort comprises 217 frontline HCP from emergency departments, infectious and pulmonary disease inpatient units, ICUs, pediatric departments and other units involved in COVID-19 patient care for weekly serologic screening for

CoV-2-specific IgG serum levels as well as symptoms of respiratory infection, work environment, and self-perceived risk. The study (DRKS00021152) is approved by local authorities (Data Security Management and Institutional Review Board of Hannover Medical School, approval \#8973_BO_K_2020). Study participants in the CoCo 1.0 cohort are HCP working at Hannover Medical School, Lower Saxony (Fig. 1) and were enrolled between 23 March and 17 April 2020. Until the end of the observational period, in total 42 SARS-CoV2-infected inpatients were treated in our hospital (more than 1000 hospital beds). In the state of Lower Saxony the total number of reported infections rose from 19 infections per 100,0000 inhabitants at the beginning on the observational period to 129 infections per 100,000 inhabitants at the end [16]. Written informed consent was obtained, participants were asked to provide blood specimens weekly during the first 2 months, followed by monthly testing. To assess the self-perceived probability of having already contracted SARS-CoV-2, the following question was asked at each visit: "How high do
SARS-CoV-2 during the first 2 months followed by monthly testing. CoCo 2.0 cohort enrolment started in May 2020 to recruit at least an additional 1000 HCP from other clinical departments of Hannover Medical School for serologic assessments every 6 months

you rate the probability of having been infected so far? (0-100\%)". Here, we report on the first 6 weeks of the CoCo 1.0 cohort.

\section{Laboratory Testing}

We used a semiquantitative ELISA for IgG based on the SARS-CoV-2 S1 spike protein domain/ receptor binding domain (Euroimmun, Lübeck, Germany) for primary testing. For additional secondary analyses in all baseline samples, positive controls, and positive or equivocal positive sera, an anti-SARS-CoV-2 S1 IgA ELISA, an anti-SARS-CoV-2 nucleocapsid protein (NCP) IgG ELISA (Euroimmun, Lübeck, Germany), and a WANTAI SARS-CoV-2 antibody rapid test (SZABO SCANDIC, Vienna, Austria-CE) were used (for more details, see supplementary material).

The neutralisation assay was performed using an in vitro-propagated SARS-CoV-2 strain isolated in Bonn, Germany, via nasopharyngeal swabbing of a patient from Heinsberg, Germany [17]. Briefly, to test SARS-CoV-2 neutralisation capacities, neutralising titers were calculated as 
the reciprocal of serum dilutions resulting in neutralisation of $50 \%$ or $90 \%$ input virus (neutralisation titers (NT) $)_{50} / \mathrm{NT}_{90}$, respectively), read out as reduction in the number of plaques (for more details, see supplementary material). The surrogate virus neutralisation test (sVNT) is described elsewhere in detail [18] and is based on the hypothesis that virus neutralising antibodies also interfere with the binding of the receptor-binding domain (RBD) of SARS-CoV-2 to surface-immobilised angiotensin-converting enzyme 2 (ACE2). In brief, hACE2 protein (Trenzyme) was coated at $300 \mathrm{mM}$ on NuncImmuno plates (Thermo Scientific) and then blocked with $2 \%$ bovine serum albumin (BSA, Sigma) and 0.1\% Tween. Then, $6 \mathrm{ng} / \mathrm{ml}$ His-tagconjugated SARS-CoV-2-S-receptor binding region (RBD) (Trenzyme) was pre-incubated (or not) with sera at different concentrations for $1 \mathrm{~h}$ at $37^{\circ} \mathrm{C}$ and added for $1.5 \mathrm{~h}$ to the ACE2-coated plates. Unbound SARS-CoV-2-S-RBD was washed off before anti-His peroxidase-labelled $\mathrm{mAb}$ (Clone 3D5) was added for $1 \mathrm{~h}$ at $37^{\circ} \mathrm{C}$. After final washing, colorimetric signal was developed by adding 3,3',5,5'-tetramethylbenzidine (Sigma) and stopped by adding $\mathrm{H}_{2} \mathrm{SO}_{4}$. Absorbance values at $450 \mathrm{~nm}$ and $570 \mathrm{~nm}$ were acquired using a SpectraMax ID3 microplate reader (Molecular Devices). Inhibition (\%) was calculated as (1 - sample optical density (OD) value/average SARS-CoV-2-S-RBD

OD value) $\times 100$.

\section{Statistical Analysis}

Data were analysed using SPSS ${ }^{\circledR}$ Statistics (version 26) and GraphPad Prism ${ }^{\circledR}$ (version 5). Data are presented as mean plus standard error of the mean (SEM) or median and range. For statistical evaluation, Pearson correlation or Fisher's exact test was performed and differences between groups were assessed by $t$ test or ANOVA with post hoc Kruskal-Wallis testing when more than two groups were compared.

\section{RESULTS}

Figure 1 shows the design of the CoCo study. The CoCo 1.0 cohort study follows 217 HCP
(65\% female) from units involved in COVID-19 patient care with longitudinal collection of biomaterials and questionnaire-based information on health status and working and living conditions. From baseline to week 6 , for a total of 1080 anti-SARS-CoV-2 IgG measurements were performed. Follow-up rates were high, with $79.8 \%$ of possible time points collected (mean 4.98 time points per HCP, range 1-7 per HCP). Of all HCP, 29.0\% reported respiratory symptoms during the 2 weeks before the first (baseline) time point with higher frequencies in men vs. women $(39.5 \%$ vs. $23.4 \%$; Fisher exact $6.3, p=0.01)$. The presence of children below the age of 12 years in the same household was associated with a higher rate of respiratory infections $(25.6 \%$ in childless households vs. $43.9 \%$ for HCP sharing a household with children under 12 years; Fisher exact 6.1, $p=0.018$ ). Among the study participants, $8.8 \%$ reported being on sick leave and 3.3\% reported having been quarantined during the 4 weeks prior to enrolment.

At baseline, $45.2 \%$ of HCP reported at least one symptom suggestive of respiratory illness, but this rate declined gradually to $25.4 \%$ by week 6 . Over the full study period, $75.6 \%$ of HCP reported at least one respiratory symptom. The rates for sick leave and quarantine over the full 6-week period were $2.8 \%$ and $2.3 \%$, respectively. Upon enrolment, $16.1 \%$ of HCP reported having been in direct contact with a confirmed SARS-CoV-2-infected person. During the period study of 6 weeks, the cumulative proportion of HCP reporting contact with confirmed infected persons was 30\%.

During this time, only $3.2 \%$ of all CoCo 1.0 cohort participants were tested for SARS-CoV-2 by polymerase chain reaction (PCR) from nasopharyngeal swabs, and all tests yielded negative results. The mean self-perceived infection probability decreased from a mean of $20.9 \%$ upon enrolment to $12.5 \%$ at week 6 $(p<0.001$, Fig. 2). This decline was evident in men and women, with women rating their risk higher than men (risk declined from $15.4 \%$ to $8.1 \%$ in men vs. from $24.1 \%$ to $14.8 \%$ in women).

As previously reported, the baseline prevalences for anti-SARS-CoV-2 S1 IgG and IgA of 


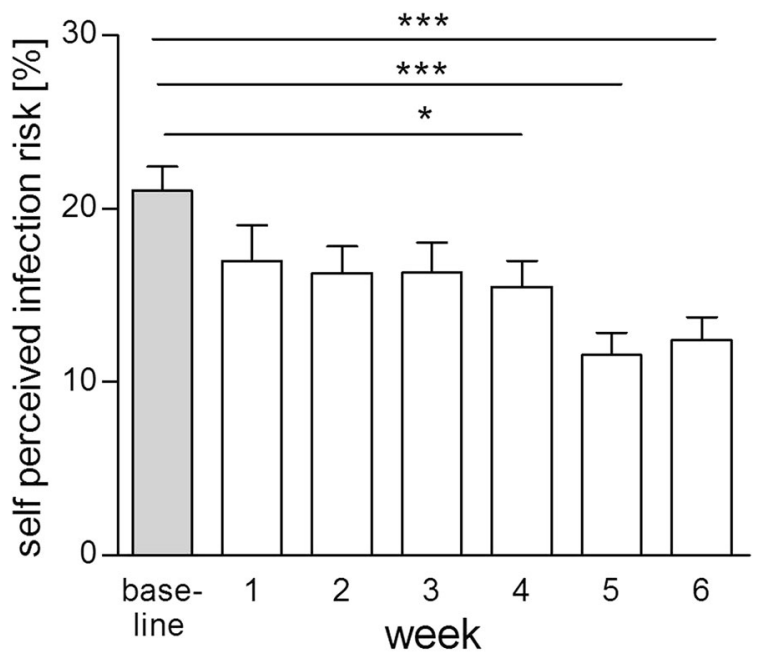

Fig. 2 Self-perceived infection risk over time. Reduction of mean self-perceived infection risk of all CoCo 1.0 cohort participants answering this question over the first 6 weeks. Bars display mean + SEM, ${ }^{*} p<0.05$, ${ }^{* * *} p<0.001$

the CoCo 1.0 cohort were low, at $0.9-1.8 \%$ and $4.1-8.7 \%$, respectively [13], and no cases of COVID-19 were observed in study participants until week 6 of observation. To assess the concordance of various testing systems in a low prevalence setting, we performed additional tests for anti-SARS-CoV-2 NCP IgG, as well as a SARS-CoV-2 antibody rapid test on the same set of samples. First, we assessed the sensitivity and specificity for all assays in convalescent patients with PCR-confirmed COVID-19. The concordance of testing results was highest between the anti-SARS-CoV-2 S1 and anti-SARS-CoV-2 NCP IgG ELISA (92.7\%, Suppl. Fig. 1). The measured levels of anti-SARS-CoV-2 S1 IgG and anti-SARSCoV-2 NCP IgG results also correlated closely (Suppl. Fig. 2A). However, their combined sensitivity within the control cohort was only $87.5 \%$ (Suppl. Fig. 1). In this group, COVID-19 severity increased with age (Suppl. Fig. 2B) and anti-SARS-CoV-2 S1 IgG ratio increased in association with COVID-19 disease severity score (Suppl. Fig. 2C).

Among participants of the CoCo 1.0 cohort, however, not one of the positive baseline results of the anti-SARS-CoV-2 S1 IgG was confirmed independently and no sample scored subsequently positive in two assays (Fig. 3). The

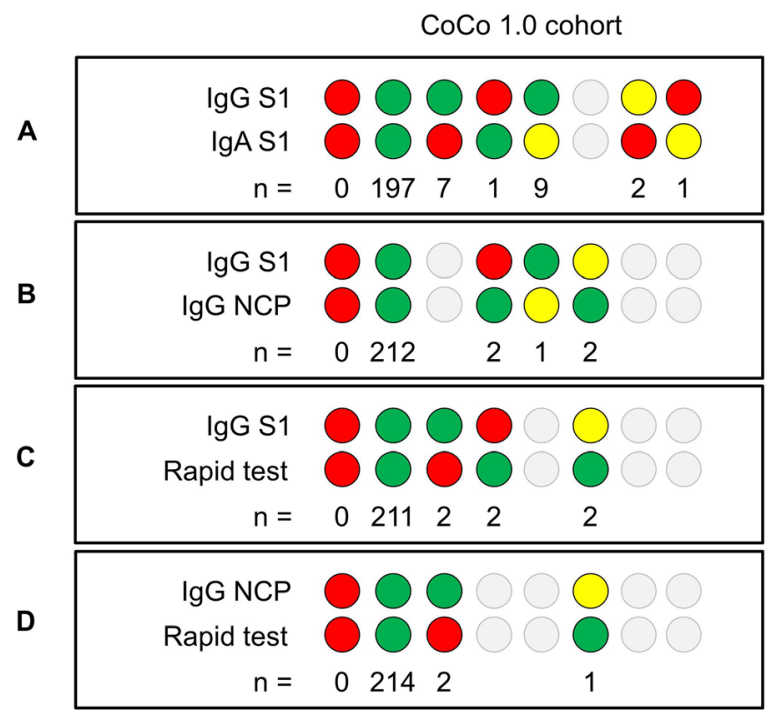

Fig. 3 Consistency of seropositivity rates of the different serological testing systems applied in CoCo 1.0 cohort. Results of the anti-SARS-CoV-2 S1 IgG versus IgA ELISA (a), anti-SARS-CoV-2 S1 IgG versus anti-SARS-CoV-2 NCP IgG ELISA (b), anti-SARS-CoV-2 S1 IgG ELISA versus the WANTAI anti-SARS-CoV-2 antibody rapid test (c), and anti-SARS-CoV-2 NCP IgG ELISA versus WANTAI anti-SARS-CoV-2 antibody rapid test (d). Red dots represent positive results (IgG ratio $>1.1$, positive band, respectively), yellow dots represent borderline positive results (IgG ratio 0.8-1.1), and green dots represent negative results (IgG ratio $<0.8$, no band, respectively)

cumulative incidence of cases with positive and borderline anti-SARS-CoV-2 S1 IgG results until week 6 of the study was $1.86 \%$ and $0.93 \%$, respectively. To account for inter-assay variability among weekly ELISAs in the CoCo 1.0 cohort and to better identify ELISA results reflecting true seroconversion, all sera (from baseline to week 6) of the eight HCP with positive or equivocal anti-SARS-CoV-2 S1 IgG/IgA ELISA results were reanalysed on a single ELISA plate (Fig. 4). OD ratios remained mostly stable, but some subjects showed declines in IgG ratios by more than 30\% over 2-3 weeks (HCP3 and 6) and others displayed oscillations in positive IgA ratios (HCP2 and 5). Remarkably, only one subject (HCP1) clearly seroconverted for both anti-SARS-CoV-2 (S1) IgG and IgA during the study period with ELISA results turning from negative to increasingly positive. Samples of HCP1 obtained at weeks 1, 2, and 4 also turned 


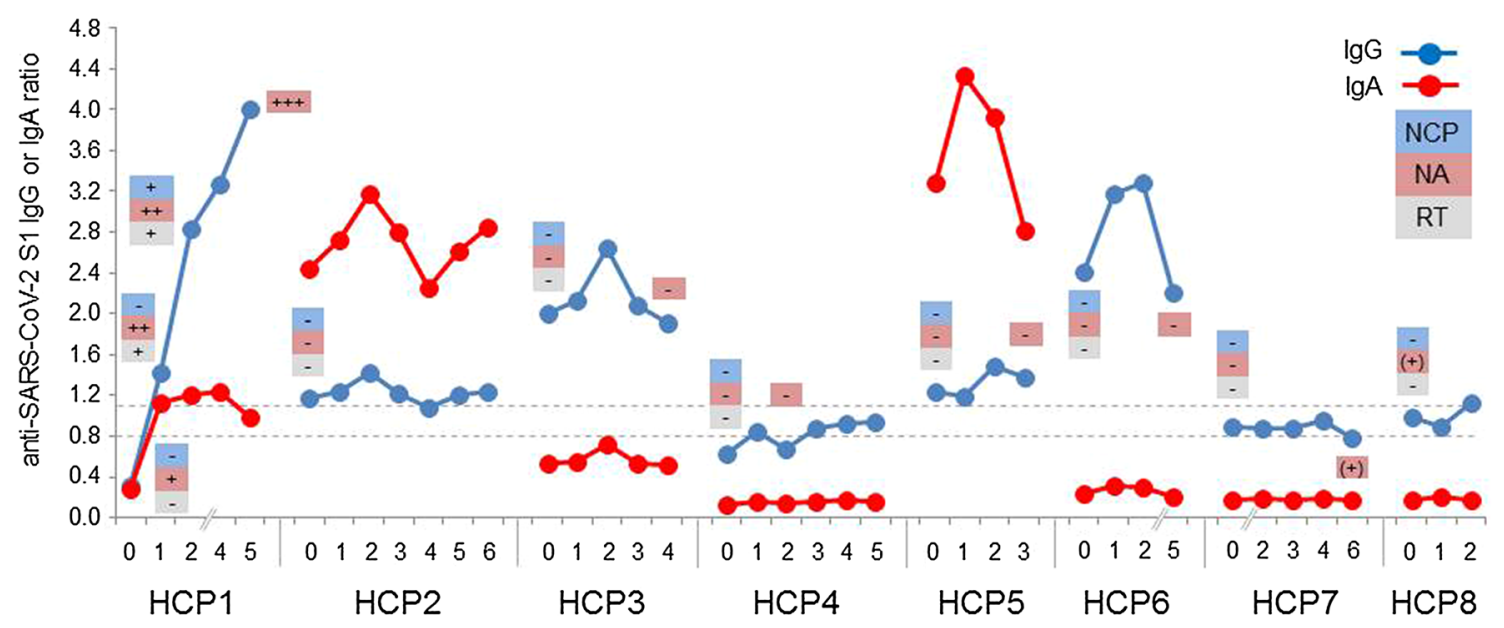

Fig. 4 Serology results of eight HCP (1-8) in the CoCo 1.0 cohort with at least one positive or borderline positive anti-SARS-CoV-2 S1 IgG ELISA during the observation period. All samples from HCP with at least one positive or borderline result at any time point (HCP 1-8) were measured on one ELISA plate. Anti-SARSCoV-2 S1 IgA is depicted in red, anti-SARS-CoV-2 S1 IgG

positive in the anti-SARS-CoV-2 NCP IgG ELISA and SARS-CoV-2 antibody rapid test (Fig. 4). HCP1 had returned from Austria prior to enrolment but reported no signs and symptoms suggestive of SARS-CoV-2 infection during the entire study period.

To assess whether the obtained positive antiSARS-CoV-2 S1 IgG serology results represented true immune responses resulting in virus neutralisation activity, we performed plaque assays using VeroE6 cells and in vitro-propagated SARS-CoV-2. Anti-SARS-CoV-2 S1 IgG results of patients with COVID-19 correlated well with $\mathrm{NT}_{90}$ neutralisation (Suppl. 3A). Except for HCP1, where longitudinal analysis was strongly suggestive of seroconversion, all HCP with positive or borderline positive anti-SARS-CoV-2 S1 IgG lacked significant neutralisation activity against SARS-CoV-2 (Fig. 4). HCP7 and HCP8 displayed very low neutralisation (1:2) in one of the two samples tested. In contrast, HCP1 developed strong SARS-CoV-2 neutralisation activity ( $\left.\mathrm{NT}_{50}, 1: 512\right)$ confirming SARS-CoV-2 immunity. Interestingly, $\mathrm{NT}_{50}$ neutralisation was detectable (1:8) before anti-SARS-CoV-2 S1 IgG and IgA results turned positive. depicted in blue. Results of anti-SARS-CoV-2 NCP (NCP), neutralisation assay (NA), or SARS-CoV-2 antibody rapid test (RT) from selected samples are indicated as positive or negative. The results of the neutralisation assay at $\mathrm{IC}_{50}$ are given as 1:2(+), 1:8 +, 1:32 and 1:64 ++, $1: 512+++$

Neutralisation assays performed using a SARS-CoV-2 variant are widely considered the gold standard, but require significant resources, safety lab requirements, and expertise. To employ a more readily applicable in vitro system, which would allow large-scale neutralisation assessments, we took advantage of a newly established surrogate viral neutralisation test (sVNT), which assesses the degree to which serum antibodies can interfere with the binding of SARS-CoV-2-S-RBD to ACE2 in vitro [18]. First, we demonstrated correlation of the sVNT data with results obtained from representative COVID-19 control samples in the plaque assay $(r=0.833, p<0.001$, Suppl. Fig. 3B). In addition, we found excellent correlation of sVNT results at $1: 180(r=0.873, p<0.001)$ and 1:540 $(r=0.945, p<0.001)$ dilutions with the ELISA anti-SARS-CoV-2 S1 IgG results (Suppl. Fig. 3C, D). The sVNT consistently identified inhibition in sera obtained from PCR-confirmed COVID19 cases, for which strong neutralisation was detected by plaque assays (Fig. 5a). Similarly, the serum of HCP1 of the CoCo 1.0 cohort showed increasing neutralisation capacity over time in the sVNT assay analogous to the plaque assay results (Fig. 5b). However, except for 


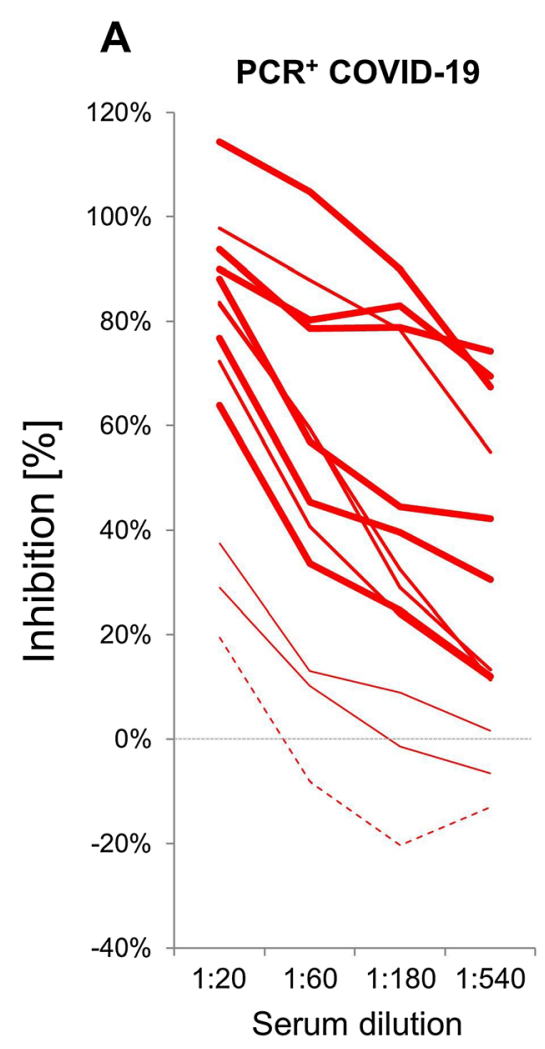

Fig. 5 Inhibition in the sVNT compared to neutralisation activity in the plaque assay. a Sera of 13 convalescent patients with PCR-confirmed COVID-19 with various neutralisation activity in the plaque assay $\left(\mathrm{IC}_{50}, 1: 16\right.$ to $\geq 1: 1024$, red lines as indicated in the legend) are depicted in according to their percent age inhibition activity in the sVNT at various dilutions as indicated. b Increase of inhibition in the sVNT during

HCP7, which at another time point showed borderline $\left(\mathrm{NT}_{50} \quad 1: 2\right)$ neutralisation signal (Fig. 4), all HCP with positive or borderline antiSARS-CoV-2 S1 IgG results had no evidence for inhibition in the sVNT (Fig. 5c).

\section{DISCUSSION}

To the best of our knowledge, this is the first prospective longitudinal study on SARS-CoV-2 functional seroconversion and self-perceived infection risk in frontline HCP. We show that in a country with comparably low COVID-19 prevalence and an advanced, resource-rich healthcare system, the current rate of anti-SARS-

\section{HCP1}

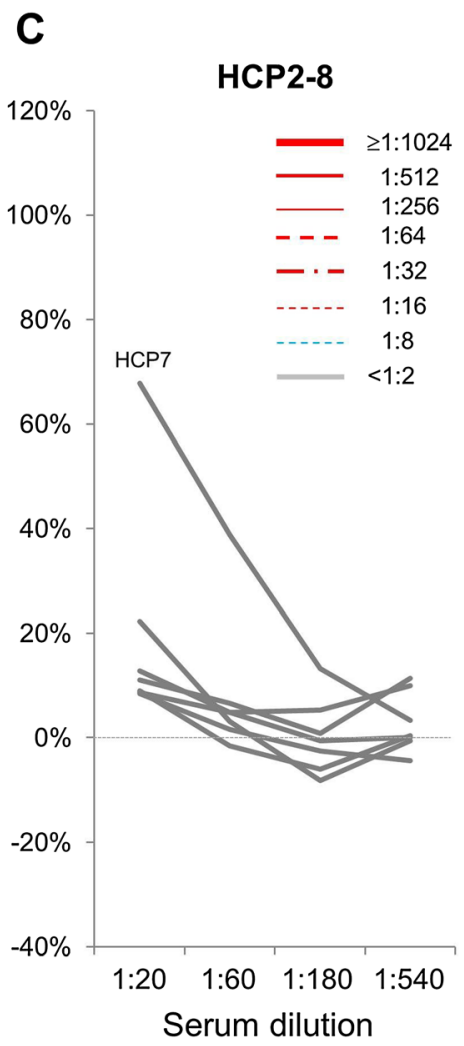

seroconversion [week (W) 1-5] of HCP1 and rise in neutralisation activity in the plaques assay as depicted by the lines. $\mathbf{c}$ Inhibition results obtained in the sVNT with sera from HCP2-8, which had least one positive or equivocal positive anti-SARS-CoV-2 S1 IgG ELISA result. None of these sera revealed significant neutralisation activity in the plaque assay $\left(\mathrm{IC}_{50} \leq 1: 2\right)$

CoV-2-Ig seroconversion in HCP after the peak of the pandemic is low, while the frequency of reported respiratory symptoms and the selfperceived risk for having contracted COVID-19 is considerable.

Since the emergence of the virus in late 2019 in China, the imminent threat to HCP of contracting COVID-19 in the workplace has been a pressing issue [19]. High rates of asymptomatic infections, ranging from $18 \%$ to $88 \%[7,20,21]$, and transmissions before the onset of symptom [22] have raised concerns about a potentially high rate of undiagnosed SARS-CoV-2 infections. In this context, serology studies are important to characterise transmission rates, 
e.g. also from children without symptoms [23], and provide insight into humoral immunity to SARS-CoV-2.

Although many uncertainties regarding COVID-19 antibody testing exist, the extent of the current crisis does not allow one to wait for "guaranteed validity" of serological diagnostics $[24,25]$, and diagnostic algorithms have to be adjusted to different settings and regional prevalences. In this regard, our study addresses several central questions about SARS-CoV-2 transmission and serological monitoring which are relevant to regions around the globe. In areas with resource-limited healthcare infrastructure and/or infection control, the pandemic has overwhelmed HCP and healthcare systems much like the initial wave of infections in Wuhan or the Lombardy region $[26,27]$. However, other countries, such as Germany, were in the fortunate position of successfully flattening the exponential spread of the virus and were able to provide hospitals and care givers with appropriate PPE in a relatively timely fashion. In spite of this, a recent national survey collecting data during the time period covered by our study reported that over $60 \%$ of German HCP, particularly women, had concerns regarding their own health while working during the current pandemic [28]. This observation is in line with our finding on self-perceived risks of having contracted SARS-CoV-2, particularly in female participants. Study participants were able to access their test results, which, as reported here, were overwhelmingly negative. This presumably contributed to the significant decline in risk perception over the study period. The high follow-up rate in the CoCo 1.0 cohort, however, supports the idea that study participants were highly interested in their personal serological status.

The observed low cumulative incidence $(0.46 \%)$ for functional anti-SARS-CoV-2 IgG is based on the combination of commercially available serology assays and in-house neutralisation tests and stands in contrast to reports from Spain, Italy and UK, in which much higher seroconversion rates in HCP are reported $[2,29,30]$. In addition to the low regional prevalence of COVID-19, sufficient access and rigorous use of PPE are likely to have contributed to this outcome. The only participant with confirmed seroconversion was most likely infected in a COVID-19 hotspot outside Germany. Interestingly, evidence for differences in the rate of hospital-acquired versus community-acquired SARS-CoV-2 infections is limited even in areas with higher COVID-19 disease burden $[2,8]$.

Our data highlight the need for a cautious approach to serology screening and result interpretation in regions with low SARS-CoV-2 infection rates. Mass testing of both symptomatic and asymptomatic HCP has been proposed to reduce spread in mild or asymptomatic cases and to protect the healthcare workforce [31]. Nevertheless, given the differences in local spread dynamics, pre-testing probabilities and targeted screening approaches must be considered [32]. The high rate and lack of discriminative value of respiratory symptoms observed in our cohort is consistent with findings from other groups [33, 34], which show that nonrespiratory symptoms in HCP (fever, anosmia/ ageusia, muscle ache, ocular pain, general malaise and extreme tiredness) were associated with positive SARS-CoV-2 PCR results, while respiratory symptoms were not. Thus, non-respiratory symptoms are likely better measures of pre-test probability in symptomatic individuals.

Our results suggest that all positive results obtained by an ELISA tests from asymptomatic individuals or those with mild or unspecific symptoms should be confirmed or disproved by an alternative serology test. Combining independent serology tests to increase diagnostic accuracy for COVID-19 may be also important when assessing unusual inflammatory disease manifestations of SARS-CoV-2 infection in selected patient groups, including children and adolescents [35]. Whether this secondary, confirmatory testing should target different SARSCoV-2 antigens or whether simply employing an alternate technique might suffice (e.g. chromatographic lateral flow rapid testing) will require further investigation. This orthogonal testing algorithm in low prevalence settings is also in line with the current recommendations for COVID-19 serology testing by the US Centers for Disease Control and Prevention (CDC) [25]. Alternatively, screening strategies may 
include parallel detection of immunoglobulins against other human coronaviruses (HKU1, OC43, NL63, and 229E) with high potential for cross-reactivity.

Studies in patients with COVID-19, ranging from mildly symptomatic to critically ill, have consistently shown that almost all patients have detectable antibodies by day 28 [31, 36]. All our study participants with PCR-confirmed COVID-19 with negative results for anti-SARSCoV-2 S1 IgG were tested at least 26 days after disease onset. Interestingly, all were female with mild disease. These factors have been suggested to be associated with weaker humoral antiSARS-CoV-2 immunity. If illness severity correlates with anti-SARS-CoV-2 IgG responses and neutralisation potency [14], we hypothesise that asymptomatic SARS-CoV-2 infection could lead to a considerable number of transient 'viral carriers' with undetectable systemic humoral immunity. These individuals would be missed by studies using serology screening only $[11,37]$. The extent to which asymptomatic cases contribute to the current pandemic is thus far unknown, and anti-SARS-CoV-2 responses in subclinical infections, as we demonstrate here, must be carefully characterised to better assess the rate of serological non-responders. The extent to which serological data can be employed to identify previously infected paucior asymptomatic persons remains unknown [5]. Of note, cellular immunity against SARS-CoV-2 alone may confer protective immunity in the absence of antibody response [37, 38].

A disadvantage of functional neutralisation assays is that they can only be performed by experienced staff in a biosafety level 3 laboratory because of the need for culture live virus. The surrogate neutralisation assay we use in this study has shown close correlation when compared to assays using live pseudotyped vesicular stomatitis virus (VSV) incorporating the $S$ protein of SARS-CoV-2 [18]. This assay consistently gave results analogous to the neutralisation assay with live SARS-CoV-2. This may become a useful tool for ascertaining robust systemic humoral immunity and assessing the kinetics of protective immunity.

Our study has several limitations. We did not perform molecular testing on respiratory specimens, which would provide information on viral carrier status in pauci- or asymptomatic HCP. We did not investigate localised immune responses, e.g. IgA in tears or mucosa fluids, or innate and cellular immune responses resulting from SARS-CoV-2 infection. Our questionnaire primarily focused on respiratory symptoms, which turned out to be of little discriminative value for identifying COVID-19. Our assessment of absolute self-perceived risk is probably a rough estimate, likely reflecting a composition of public and individual risk perception. Of note, this report represents an interim analysis, and the further CoCo cohort recruitment will likely provide more information on these topics.

\section{CONCLUSION}

Our data show a low functional seroconversion rate in HCP, contrasting with a considerable self-perceived infection probability. Self-reported respiratory symptoms appear to be too unspecific to inform pre-test probability and serology test result interpretation. Our data highlight the need for a cautious approach to serology screening and result interpretation in regions with low SARS-CoV-2 infection rates. For analyses of humoral SARS-CoV-2-specific immune response in a low pre-test probability setting, positive results from single measurements should be confirmed by alternative serology tests or functional assays.

\section{ACKNOWLEDGEMENTS}

We thank all participants of the CoCo study. Also, we would like to thank Marion Hitzigrath, Melanie Ignacio, Annkathrin Anton, Till Redeker, Madeleine Rommel, Luis Manthey, and Nele Stein for technical and logistical support. We thank the CoCo study team for help with enrolment and blood collection for the Coco cohort (Martina Toussaint, Marcel Winkelmann, Mark Greer, Marcus Bachmann, and Birgit Heinisch). 
Funding. This study is supported by an unrestricted grant from Novartis to GMNB, AJ, $\mathrm{DE}, \mathrm{CH}$, from PARI to $\mathrm{CH}, \mathrm{AJ}, \mathrm{CD}, \mathrm{MW}$, and Verein Kinderherz Hannover. GMNB, MW and AJ are supported by the German Centre for Infection Research (DZIF). RF is supported by Deutsche Forschungsgemeinschaft, DFG Excellence Strategy-EXC 2155 "RESIST"-project number $39,087,428$, Sonderforschungsbereich SFB900 project number 158989968-SFB 900 and by a fund of the state of Lower Saxony (1476103-184 CORONA-11/20). The funders had no role in study design, data collection and analysis, decision to publish, or preparation of the manuscript. The rapid service fee is funded by the unrestricted grant from Novartis.

Authorship. All named authors meet the International Committee of Medical Journal Editors (ICMJE) criteria for authorship for this article, take responsibility for the integrity of the work as a whole, and have given their approval for this version to be published.

Authorship Contribution. GMNB, CH, AJ designed and managed the project, analysed the data and drafted the manuscript. AC managed study enrolment, designed the workflow and questionnaires, organised data collection and biobanking, and analysed the data. MS performed the ELISA and rapid test experiments and designed the laboratory workflow. RF and BB developed the sVNT and BB and SW performed the inhibition experiments; HS and BS performed and analysed the neutralisation assays. TT, TG, MZK, A-LB, ATT, AZS, AH, CD, MW, IP, TW, and DE supported recruitment, blood sampling and follow-up visits. All authors discussed the results and approved the final manuscript.

Disclosures. Alexandra Jablonka reports grants and personal fees from Novartis, grants and personal fees from Abbvie, grants and personal fees from Gilead, personal fees from Roche, outside the submitted work; Torsten Witte reports grants and personal fees from Novartis, grants and personal fees from Abbvie, personal fees from Gilead, personal fees from Chugai, personal fees from Sanofi-Aventis, non- financial support from Aesku.Diagnostics, outside the submitted work; Diana Ernst reports grants and personal fees from Novartis, grants and personal fees from Abbvie, grants and personal fees from Gilead, personal fees from Sanofi Aventis, personal fees from GSK, outside the submitted work; Isabell Pink reports personal fees from Chiesi and personal fees from Boehringer Ingelheim, outside the submitted work; Georg M.N. Behrens reports grants and personal fees from Gilead, and personal fees from ViiV Healthcare, MSD, and Janssen outside the submitted work. Anne Cossmann, Metodi V. Stankov, Bianca Schulte, Hendrik Streeck, Reinhold Förster, Berislav Bosnjak, Stefanie Willenzon, Anna-Lena Boeck, Anh Thu Tran, Thea Thiele, Theresa Graalmann, Moritz Z. Kayser, Anna Zychlinsky Scharff, Christian Dopfer, Alexander Horke, Martin Wetzke and Christine Happle have nothing to disclose.

Compliance with Ethics Guidelines. The study (DRKS00021152) is approved by local authorities (Data Security Management and Institutional Review Board of Hannover Medical School, approval \#8973_BO_K_2020). Written informed consent was obtained.

Data Availability. The datasets generated during and/or analyzed during the current study are available from the corresponding author on reasonable request.

Open Access. This article is licensed under a Creative Commons Attribution-NonCommercial 4.0 International License, which permits any non-commercial use, sharing, adaptation, distribution and reproduction in any medium or format, as long as you give appropriate credit to the original author(s) and the source, provide a link to the Creative Commons licence, and indicate if changes were made. The images or other third party material in this article are included in the article's Creative Commons licence, unless indicated otherwise in a credit line to the material. If material is not included in the article's Creative Commons licence and your intended use is not permitted by statutory regulation or exceeds the permitted use, you will need to obtain 
permission directly from the copyright holder. To view a copy of this licence, visit http:// creativecommons.org/licenses/by-nc/4.0/.

\section{REFERENCES}

1. Rosenbaum L. Facing Covid-19 in Italy-ethics, logistics, and therapeutics on the epidemic's front line. N Engl J Med. 2020;382(20):1873-5.

2. Houlihan NVC, Byrne T, Lewer D, et al. SARS-CoV-2 virus and antibodies in front-line health care workers in an acute hospital in London: preliminary results from a longitudinal study. 2020. https://www.medrxiv.org/content/101101/202006 0820120584v1article-info. Accessed 31 July 2020.

3. Centers for Disease Control and Prevention. Cluster of severe acute respiratory syndrome cases among protected health-care workers-Toronto, Canada, April 2003. MMWR Morb Mortal Wkly Rep. 2003;52(19):433-6.

4. Memish ZA, Zumla AI, Assiri A. Middle East respiratory syndrome coronavirus infections in health care workers. N Engl J Med. 2013;369(9):884-6.

5. Treibel TA, Manisty C, Burton M, et al. COVID-19: PCR screening of asymptomatic health-care workers at London hospital. Lancet. 2020;395(10237): 1608-10.

6. Kimball A, Hatfield KM, Arons M, et al. Asymptomatic and presymptomatic SARS-CoV-2 infections in residents of a long-term care skilled nursing facility-King County, Washington, March 2020. MMWR Morb Mortal Wkly Rep. 2020;69(13): 377-81.

7. Mizumoto K, Kagaya K, Zarebski A, Chowell G. Estimating the asymptomatic proportion of coronavirus disease 2019 (COVID-19) cases on board the Diamond Princess cruise ship, Yokohama, Japan, 2020. Euro Surveill. 2020;25(10):2000180.

8. Steensels D, Oris E, Coninx L, et al. Hospital-wide SARS-CoV-2 antibody screening in 3056 staff in a tertiary center in Belgium. JAMA. 2020;324:195-7.

9. Pan A, Liu L, Wang C, et al. Association of public health interventions with the epidemiology of the COVID-19 outbreak in Wuhan, China. JAMA. 2020;323:1-9.

10. Folgueira M-RM, Alonso-Lopez M, et al. SARS-CoV2 infection in Health Care Workers in a large public hospital in Madrid, during March 2020. 2020. https://www.medrxiv.org/content/101101/2020040 720055723v2. Accessed 7 July 2020.

11. Long QX, Liu BZ, Deng HJ, et al. Antibody responses to SARS-CoV-2 in patients with COVID-19. Nat Med. 2020;26(6):845-8.

12. Cheng MP, Yansouni CP, Basta NE, et al. Serodiagnostics for severe acute respiratory syndrome-related coronavirus-2: a narrative review. Ann Intern Med. 2020. https://doi.org/10.7326/M20-2854.

13. Behrens GMN, Cossmann A, Stankov MV, et al. Perceived versus proven SARS-CoV-2-specific immune responses in health-care professionals. Infection. 2020;48:631-4.

14. Robbiani DF, Gaebler C, Muecksch F, et al. Convergent antibody responses to SARS-CoV-2 in convalescent individuals. Nature. 2020;584:437-42.

15. Sethuraman $\mathrm{N}$, Jeremiah SS, Ryo A. Interpreting diagnostic tests for SARS-CoV-2. JAMA. 2020;323(22):2249-51.

16. RKI. COVID-19 in Germany. 2020. https://www.rki. de/DE/Content/InfAZ/N/Neuartiges_Coronavirus/ Fallzahlen.html. Accessed 31 July 2020.

17. Streeck H SB, Kuemmerer B, et al. Infection fatality rate of SARS-CoV-2 infection in a German community with a super-spreading event. 2020. https:// www.medrxiv.org/content/101101/ $2020050420090076 v 2$. Accessed 7 July 2020.

18. Bošnjak B SS, Willenzon S, et al. Low levels of neutralizing anti-SARS-CoV-2-S antibodies in sera of convalescent COVID-19 patients with mild clinical symptoms. 2020. https://doi.org/10.1101/ 2020.07.12.20151407.

19. Lai J, Ma S, Wang Y, et al. Factors associated with mental health outcomes among health care workers exposed to coronavirus disease 2019. JAMA Netw Open. 2020;3(3):e203976.

20. Day M. Covid-19: four fifths of cases are asymptomatic, China figures indicate. BMJ. 2020;369: $\mathrm{m} 1375$.

21. Sutton D, Fuchs K, D'Alton M, Goffman D. Universal screening for SARS-CoV-2 in women admitted for delivery. N Engl J Med. 2020;382(22): 2163-4.

22. Bai Y, Yao L, Wei T, et al. Presumed asymptomatic carrier transmission of COVID-19. JAMA. 2020;323: 1406-7.

23. Buonsenso D, Zampino G, Valentini P. Novel coronavirus disease 2019 infection in children: the 
dark side of a worldwide outbreak. Front Pediatr. 2020;8:215.

24. Weinstein MC, Freedberg KA, Hyle EP, Paltiel AD. Waiting for certainty on Covid-19 antibody testsat what cost? N Engl J Med. 2020;383(6):e37.

25. CDC. Interim guidelines for COVID-19 antibody testing. 2020. https://www.cdc.gov/coronavirus/ 2019-ncov/lab/resources/antibody-tests-guidelines html. Accessed 31 July 2020.

26. Sheehan MC, Fox MA. Early warnings: the lessons of COVID-19 for public health climate preparedness. Int J Health Serv. 2020;50(3):264-70.

27. Bressan S, Buonsenso D, Farrugia R, et al. Preparedness and response to pediatric CoVID-19 in European emergency departments: a survey of the REPEM and PERUKI networks. Ann Emerg Med. 2020 . https://doi.org/10.1016/j.annemergmed. 2020.05.018.

28. Paffenholz P, Peine A, Hellmich M, et al. Perception of the 2020 SARS-CoV-2 pandemic among medical professionals in Germany: results from a nationwide online survey. Emerg Microbes Infect. 2020;9: 1590-9.

29. Folgueira MD, Alonso-Lopez MA, Delgado R. SARSCoV-2 infection in health care workers in a large public hospital in Madrid, Spain, during March 2020. 2020. https://www.medrxiv.org/content/ $101101 / 2020040720055723 v 2$. Accessed 31 July 2020.

30. Garcia-Basteiro AL, Moncunill G, Tortajada M, et al. Seroprevalence of antibodies against SARS-CoV-2 among health care workers in a large Spanish reference hospital. Nat Commun. 2020;11(1):3500.

31. Black JRM, Bailey C, Przewrocka J, Dijkstra KK, Swanton C. COVID-19: the case for health-care worker screening to prevent hospital transmission. Lancet. 2020;395(10234):1418-20.

32. Watson $\mathrm{J}$, Whiting $\mathrm{PF}$, Brush JE. Interpreting a covid-19 test result. BMJ. 2020;369:m1808.

33. Tostmann A, Bradley J, Bousema T, et al. Strong associations and moderate predictive value of early symptoms for SARS-CoV-2 test positivity among healthcare workers, the Netherlands, March 2020. Euro Surveill. 2020;25(16):2000508.

34. Lan FY, Filler R, Mathew S, et al. COVID-19 symptoms predictive of healthcare workers' SARS-CoV-2 PCR results. PLoS One. 2020;15(6):e0235460.

35. Feldstein LR, Rose EB, Horwitz SM, et al. Multisystem inflammatory syndrome in US children and adolescents. N Engl J Med. 2020;383(4):334-46.

36. Wolfel R, Corman VM, Guggemos W, et al. Virological assessment of hospitalized patients with COVID-2019. Nature. 2020;581(7809):465-9.

37. Braun J, Loyal L, Frentsch M, et al. SARS-CoV-2reactive $T$ cells in healthy donors and patients with COVID-19. Nature. 2020. https://doi.org/10.1038/ s41586-020-2598-9.

38. Mateus J, Grifoni A, Tarke A, et al. Selective and cross-reactive SARS-CoV-2 T cell epitopes in unexposed humans. Science. 2020. https://doi.org/10. 1126/science.abd3871. 